\section{Élasticité du centromère}

Jean-Pierre Labbé
$>$ Outre l'activité du fuseau mitotique et des protéines qui lui sont associées, les chromosomes eux-mêmes jouent un rôle important dans leur ségrégation. La séparation des chromatides nécessite la dégradation par la séparase de protéines particulières, qui agissent comme une glu formée par un complexe protéique: la cohésine. Le complexe cohésine, très conservé, est nécessaire à l'établissement et à la maintenance des chromatides à l'intérieur d'une structure en « anneau ». Ce modèle de l'anneau suggère que la cohésine posséderait une grande mobilité quand elle est associée à la chromatine. II semble, toutefois, que la cohésine ne peut pas, seule, résister aux forces exercées par les microtubules, et doit être renforcée par une force de cohésion provenant de I'ADN. De fait, la région intérieure du centromère se comporte comme un élément élastique, et il a été montré que la titine était un composant chromosomique: d'où l'hypothèse selon laquelle elle permettrait au chromosome d'être élastique et de résister à la cassure pendant la mitose, hypothèse appuyée par le fait que la déformabilité et la rigidité des courbures chromosomiques sont en accord avec le modèle décrit pour l'élasticité de la titine. <
Le centromère des mammifères - domaine à multiples fonctions dont la structure reflète la complexité [1] est essentiel au passage des chromosomes dans les cellules filles pendant la division cellulaire. Défini comme une entité cytogénétique, il est la constriction primaire qui sert au chromosome de site d'attachement au fuseau mitotique.

\section{Organisation structurale et fonctionnelle $\mathrm{du}$ centromère}

L'hétérochromatine est associée au niveau du centromère à une structure protéique importante, le kinéto-

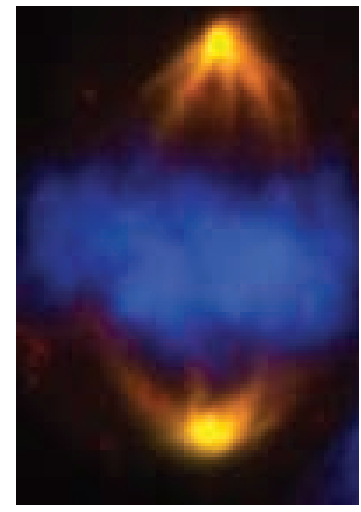

chore [2], structure trilamellaire située à

la surface extérieure du centromère. Le kinétochore interagit directement avec les microtubules du faisceau mitotique et joue un rôle primordial dans la production des forces nécessaires au mouvement des chromosomes pendant la division cellulaire. II est constitué de trois régions, un plateau interne, entourant un domaine central dont l'ADN est constitué de séquences répétées, et un domaine d'appariement représentant le site d'interaction entre les chromatides sœurs. La cohésion des chromatides comme leur séparation sont des phénomènes précisément réglés qui dépendent de la qualité de l'association entre les chromatides sœurs. L'existence d'une tension est fondamentale dans la stabilisation de l'attachement des chromatides sœurs; des images électroniques ont montré que la région centrale du chromosome est résistante à la déformation [3]. Le centromère organise sa propre ségrégation, en mitose comme en méiose, qui est différente de celle des bras du chromosome. Les centromères des chromosomes mitotiques se séparent avec une chronologie précise, les acrocentriques étant les derniers à se séparer [4]. La notion de division prématurée centromérique $(\mathrm{dpc})$, introduite par H. Fitzgerald en 1975 [5], décrit la division prématurée du chromosome $X$ en comparaison de celle des autres chromosomes observée au cours de la même métaphase. La génétique du dpc n'est pas totalement comprise. II a été proposé que ces anomalies de $\mathrm{dpc}$ du chromosome $X$ soient responsables de certains cas de trisomie 21 et soient associées à un grand nombre de maladies comme le syndrome de Robert [6]. Cette 
séparation prématurée est la manifestation d'une instabilité chromosomique. À la métaphase, la distance précise séparant les centromères de chromatides sœurs, qui permet d'évaluer objectivement la qualité de la séparation, peut être modifiée [7] ; l'acide okadaïque, un inhibiteur des phosphatases $l$ et $2 A$, entraîne une anomalie dans la séparation des chromatides à la transition métaphase/anaphase et la formation de diplochromosomes dans des cellules Hela [8].

L'architecture moléculaire et la fonction dynamique du complexe centromère-kinétochore ne sont étudiées que depuis quelques années. La façon dont la chromatine est assemblée au sein du centromère métaphasique ne peut être comprise que dans un modèle structural particulier (Figure 1A). Le centromère sensible à la 5-azacytidine et au Hoetsch 33258 par modification de la condensation a un diamètre de $100 \mathrm{~nm}$; en l'absence de ces agents chimiques, il retrouve sa morphologie initiale. Cela peut mimer la configuration in vivo du centromère interphasique [10].

Plusieurs classes de protéines qui pourraient influencer la conformation du centromère ont été mises en évidence, qui semblent spécifiques. Les plus connues sont les auto-antigènes Cenp $A$ (centromere protein A) (17 kDa), Cenp B (80 kDa) et Cenp C (140 kDa). Cenp G (95 kDa) a été détectée associée avec la séquence $\alpha 1$ du centromère, tandis que Cenp $\mathrm{H}$ a été détectée au sein du plateau interne du kinétochore. La protéine Cenp $B$, plus particulièrement centromérique, n'est pas nécessaire puisqu'elle n'est pas présente dans le chromosome $y$ qui ségrège pourtant normalement. Les Incenp (inner centromere proteins) [11], qui interagissent directement avec la tubuline, sont concentrées au niveau du centromère pendant la prométhaphase. Les Clips [11] sont, quant à elles, localisées dans une structure filamenteuse qui part du domaine central et s'étend entre les centromères des chromatides. Une famille de protéines SMC (structural maintenance of chromosomes), qui sont des ATP binding cassettes, est nécessaire à la condensation du chromosome et à la disjonction des chromatides [12].

II a été montré, par microscopie électronique, en utilisant comme sonde nées (d'après [2]).
Cenp $B$, que l'élongation du domaine $\alpha$-satellite est différente de l'étirement du kinétochore et que la région centrale du chromosome résiste à la déformation imposée par les microtubules. Cette élongation, qui commence en prométaphase, atteint son niveau maximal à la métaphase; l'élongation du domaine $\alpha$-satellite riche en Cenp B représente le dernier stade dans l'élaboration structurale du centromère.

L'attachement bipolaire des chromosomes aux microtubules fait intervenir des forces orientées qui créent des tensions dans le centromère et dans la chromatine centromérique [13]. II a été montré que l'anaphase ne débutait que lorsque tous les kinétochores étaient attachés aux microtubules [14]. Ces tensions peuvent être réduites par le Taxol ${ }^{\circledR}$, qui inhibe la liaison kinétochore-microtubule. Le centromère jouerait ainsi le rôle d'un tensiomètre moléculaire, travaillant au centre de l'appareil mitotique afin de convertir la force mécanique en signal biologique [15]: il existe ainsi une importante corrélation entre la force mécanique et la chimie du centromère, montrée par les analyses des phosphoprotéines associées au kinétochore [16]. Une hypothèse importante formule que les protéines contenues dans le centromère, dont les conformations sont directement affectées par le stress mécanique, induisent des modifications dans diverses activités enzyma-

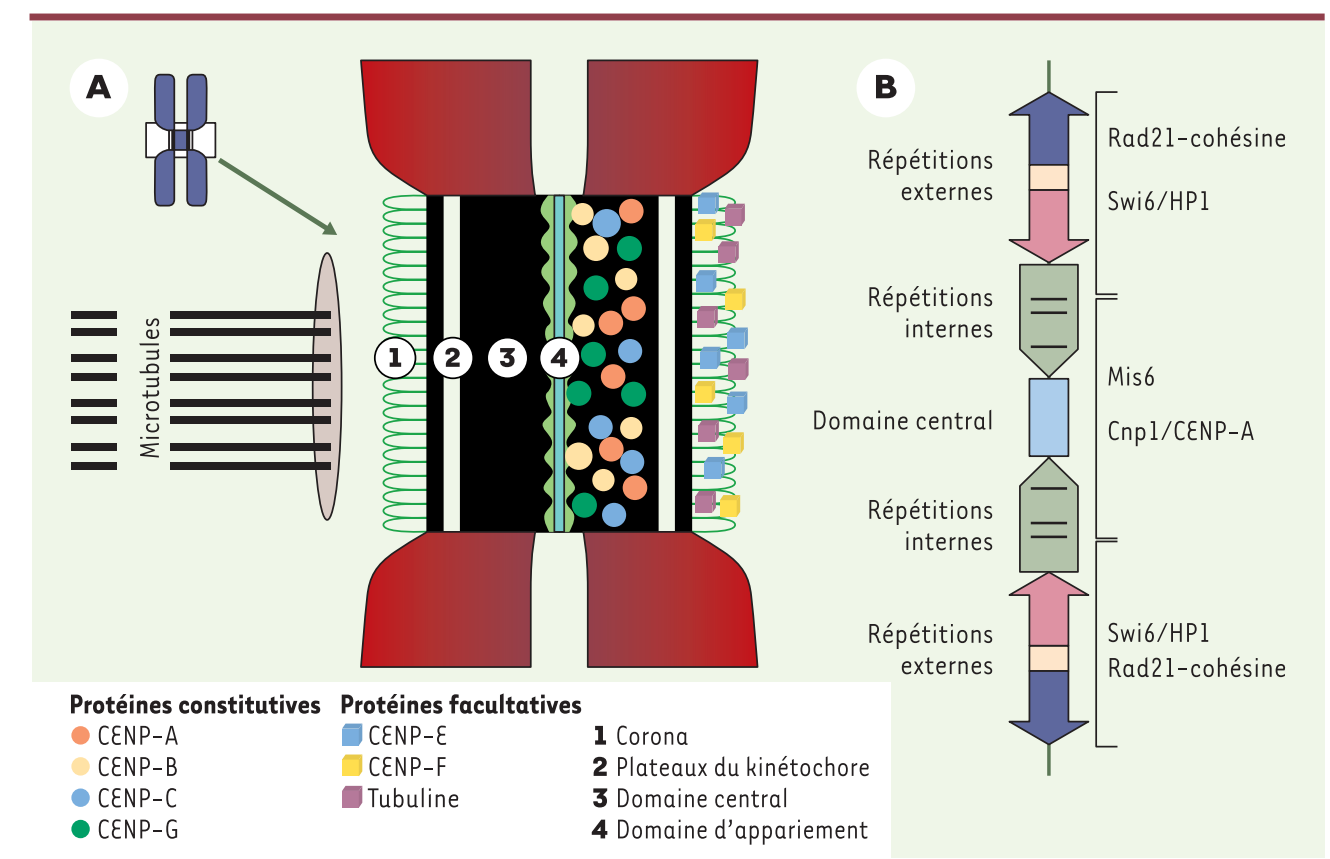

Figure 1. Modèles de centromère. A. Modèle de centromère humain : on y voit les protéines associées, ainsi que la position des microtubules exerçant des forces sur le kinétochore (d'après [9]). B. Modèle de centromère de Schizosaccharomyces pombe: les protéines associées au domaine central et celles associées aux répétitions externes qui règlent l'association du complexe Rad 21-cohésine avec les régions péricentriques sont mention- 
tiques ou d'interactions [13]. La chromatine centromérique pourrait, quant à elle, fournir un environnement reproductible aux mécanismes tensiométriques [15]. Les complexes macromoléculaires sont dépendants de l'extension de l'hétérochromatine composée de séquences satellites. La chromatine $\alpha$-satellite se comporte comme un élastique pendant la mitose et des extensions réversibles de 200 à 400 nm ont été observées. Cenp $B$, non représentée dans le centromère $d u$ chromosome $Y$, est exclue de ce rôle. En interaction avec Cenp C, elle serait plutôt impliquée dans l'assemblage de la structure kinétochore-centromère.

Ces événements moléculaires se produisent au sein d'un complexe macromoléculaire dominé par l'hétérochromatine, qui fournit le lien matériel entre les chromatides sœurs. Cependant, la nature exacte de ce complexe n'est pas encore élucidée: implique-t-il seulement le complexe cohésine, ou une association de la cohésine avec des protéines importantes de la contractilité?

\section{Attachement des chromatides sœurs et cohésine}

La cohésion [17, 18] entre les chromatides sœurs s'oppose aux forces de cisaillement exercées par les microtubules; la perte de cette cohésion permet la séparation postérieure des chromatides sœurs pendant l'anaphase (Figure 2). Cet attachement est, durant la plus grande partie du cycle cellulaire, dû à la cohésine $[19,20]$, un complexe protéique formé de quatre sousunités: deux protéines ATP binding cassettes, membres des SMC (SMCl, SMC3), et deux protéines non-SMC: SCCl (sister chromatid cohesine 1) et SSC3. Ainsi, I'alignement du chromosome pendant la métaphase ne dépend pas seulement du cisaillement des forces exercées par les microtubules ou le kinétochore, mais aussi de l'opposition aux forces dites de cohésion exercées par la cohésine. La déplétion en cohésine provoque une séparation prématurée des chromatides sœurs.

Un modèle du complexe « en anneau » a été proposé [21, 22], dans lequel la cohésine maintient les chromatides sœurs ensemble à l'intérieur de cet anneau (Figure $3 B$ ); cet anneau pourrait également se lier fortement avec les chromatides sœurs (Figures $3 \mathrm{~A}$ et $3 \mathrm{C}$ ). Ce modèle suggère que la cohésine possède une très grande mobilité quand elle est associée à la chromatine. Toutes les têtes des sous-unités motrices SMCl et SMC3 possèdent des séquences impliquées dans la fonction ATPase. SMCl et SMC3 forment, par leur domaine central, un hétérodimère en « V » qui s'associe avec les sous-unités $\mathrm{SCCl}$ et SCC3. L'association de l'hétérodimère avec les deux extrémités de SCCl forme alors un large anneau qui entoure les (ou se lie aux) chromatides sœurs. La ségrégation survient lors de la coupure, par la séparase, de la sous-unité $\mathrm{SCCl}$ en fragments amino- et carboxyterminaux, ce qui conduit à une dissociation des éléments de l'anneau. En termes de régulation, la séparase est inactivée par son association avec la sécurine. C'est l'ubiquitinylation de la sécurine par une $\varepsilon 3$ ligase, l'anaphase promoting complex/cyclosome (APC/C), et par son activateur $\mathrm{CdC20}$ qui permet son ciblage pour sa destruction par la protéase 26S, libérant ainsi une séparase active. Ce n'est toutefois pas la seule voie de contrôle du clivage de la cohésine.

La cohésine se fixe plus fortement au niveau du centromère que dans le reste des chromatides. Des séquences particulières CAR (cohesine associated region), très riches en AT, sont des lieux privilégiés d'interaction avec la cohésine. Dans la plupart des organismes, la cohésine est concentrée dans les régions centromériques et maintenue jusqu'à l'anaphase. Les centromères de Schizosaccharomyces pombe sont de bons modèles d'étude des centromères d'eucaryotes supérieurs. Ils sont composés de grands blocs de répétitions inversées et d'un domaine central. Ce dernier est entouré par des répétitions internes (imr), puis externes (otr). Les gènes de cette chromatine sont faiblement réprimés dans le domaine central et fortement réprimés dans les répétitions inversées. L'association avec différentes protéines contrôle les régions silencieuses du centromère: les protéines de levure Swi6 (HPl chez la drosophile) et Chpl sont associées aux répétitions externes, tandis que les protéines Mis6 et Cnpl (homologue de la protéine humaine Cenp A) sont 
associées aux répétitions internes et au domaine central (Figure 1B). Des gènes d'ARNt marquent la zone de transition entre ces domaines distincts. De récentes expériences ont montré que la distribution de la cohésine n'était pas homogène: on note une quantité 6 fois plus élevée de cohésine associée aux régions centromériques que dans les quelques sites répartis le long des bras. Swi6 ( $\mathrm{HPl}$ ), nécessaire à l'association du complexe Rad 21 ( $\mathrm{SCCl}$ )-cohésine avec la région péricentrique du centromère, recrute la cohésine pour la région péricentrique mais pas le long des bras du chromosome.

Si la dégradation/dissociation de la cohésine chez S. pombe est globale, elle survient en plusieurs étapes chez les métazoaires. Durant la prophase-métaphase, la cohésine est dissociée du chromosome indépendamment de l'intervention du complexe APC/C: c'est le stade prophase dissolution pathway (PDP). La cohésine qui reste fixée au centromère est par la suite dégradée à l'anaphase par I'APC/C. La bi-orientation des chromatides sœurs lors de la transition prophase/métaphase, c'est-à-dire leur attachement, via les kinétochores, aux microtubules émanant des pôles opposés du fuseau, n'est possible que par leur forte cohésion entre elles et par la quantité importante de cohésine fixée dans la région péricentrique entourant le complexe centromère-kinétochore. Cela montre bien l'influence du centromère sur la cohésion des chromatides sœurs.

L'hétérochromatine péricentrique [23] n'est donc pas seulement un environnement favorable à l'assemblage protéique du kinétochore, mais le lieu privilégié de la fixation de la cohésine. Le centromère est ainsi le site d'assemblage du complexe protéique du kinétochore, qui permet l'attachement et les mouvements des chromosomes le long du fuseau mitotique, et dont la cohésion péricentrique a pour fonction de résister aux forces imposées par les microtubules. Mais le complexe centromèrekinétochore joue également le rôle d'activateur de l'association de la cohésine avec la chromatine péricentrique. Des modèles ont été proposés pour rendre compte de cette fonction majeure de la cohésine dans la résistance aux forces exercées par les microtubules. Cependant, aucun ne paraît vraiment satisfaisant, dans la mesure où la cohésine, in vitro, ne semble pas capable de se fixer aussi fortement à I'ADN et de résister aux forces de cisaillement exercées par les microtubules [24]. D'où l'idée qu'un autre complexe multiprotéique pourrait jouer un rôle conjoint dans l'élasticité du centromère, dépendant de la titine.

\section{Élasticité et titine}

Les chromosomes, très élastiques, peuvent être étirés dans leur longueur [25]. La microscopie confocale in situ révèle que c'est le centromère qui subit la déformation de son domaine central. Une étude de la déformabilité des chromosomes [26] montre qu'ils reviennent à leur forme primitive après avoir été étirés 10 fois en longueur. Pour un étirement de l'ordre de 10 à 100 fois, ils se transforment en filaments fins et, au-delà, il y a cassure au niveau du centromère.

Chaque chromosome a une structure très ordonnée et élastique maintenant son intégrité tout au long de la division cellulaire. L'ADN chromosomique doit être compacté de manière à s'insérer dans le noyau qui mesure 5 à $20 \mu \mathrm{m}$; les chromosomes sont, quant à eux, compactés 5 à 10 fois à la mitose. Plusieurs modèles ont été proposés pour la structure du chromosome mitotique: formation d'un simple solénoïde, formation d'un gel non organisé ou attachement des boucles de chromatine à un réseau central. Les mesures du module de young (facteur entre force et déformation) montrent l'existence d'un gel polymère; les mesures de flexibilité, en analysant les fluctuations de courbure, suggèrent que les chromosomes sont formés d'un ou de plusieurs axes rigides, minces, élastiques, dont on peut estimer le diamètre inférieur à $20 \mathrm{~nm}$, entourés d'une enveloppe

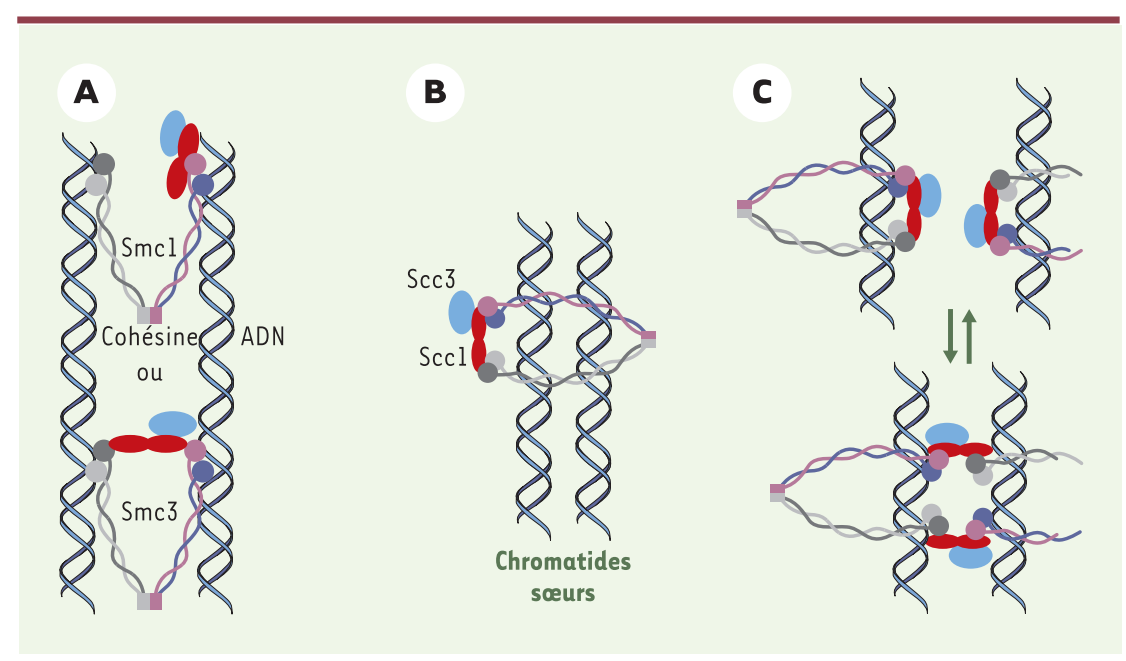

Figure 3. Modèles de cohésion des chromatides sceurs. A. Protéine SMC (structural maintenance of chromosome) interagissant directement avec l'ADN (d'après [12] et [22]). B. Le modèle de C.H. Haering et K. Nasmyth [20], en anneau, montre les deux chromatides sœurs enlacées par un seul complexe cohésine. C. Modèle double-ring: chaque chromatide est associée à une molécule de complexe cohésine, la cohésion entre chromatides étant obtenue par un changement dans l'association avec SCCl (sister chromatid cohesine), par des interactions intracomplexes (en haut) ou intercomplexes (en bas) (d'après [22]). SMCl: en violet; SMC3: en gris; SCCl: ovale rouge; SCC3: ovale bleu. 
souple. Les propriétés de ces axes correspondent aux modèles développés pour l'élasticité de molécules semblables à la titine [27]. Les caractéristiques de la titine sont retrouvées au niveau de l'axe du chromosome après chaque cycle de déformation. Elle contrôlerait ainsi le diamètre axial des chromosomes mitotiques, comme elle contrôle la longueur des filaments fins du sarcomère. Des anticorps anti-titine, extraits de sérums de malades contenant des anticorps autoimmuns (scleroderma), reconnaissent un épitope situé sur le chromosome mitotique condensé de cultures de cellules humaines ou d'embryon de drosophile [28]. De même, des anticorps anti-énolase réagissent avec le kinétochore (la titine et l'énolase [enzyme glycolytique] sont les deux protéines spécifiques du sarcomère les plus précocement exprimées au cours de la myogenèse). Les phénotypes de mutants de $D$-titine suggèrent un rôle identique pour la titine à la fois dans le muscle et dans la structure et l'élasticité du chromosome. Une mutation dans la D-titine provoque une condensation défectueuse, une cassure dans le chromosome, une perte de diploïdie et une séparation prématurée des chromatides [29]. Un nouveau modèle de la structure chromosomique fondé sur des mesures d'élasticité et la capacité d'être déformé longitudinalement peut donc être proposé, qui implique la titine: le chromosome condensé ou mitotique est formé par un ou plusieurs axes composés de molécules de titine, entourés d'une enveloppe souple de chromatine (Figure 4) $[27,30,31]$.

Dans ce contexte du chromosome, les partenaires habituels de la titine sont-ils présents? Des résultats, parfois remis en cause, montrent que l'actine nucléaire peut intervenir dans le remodelage de la chromatine lors de la transcription et qu'elle est l'un des composants de la matrice nucléaire sous forme de monomères ou de petits oligomères courts. In vivo, on montre, par

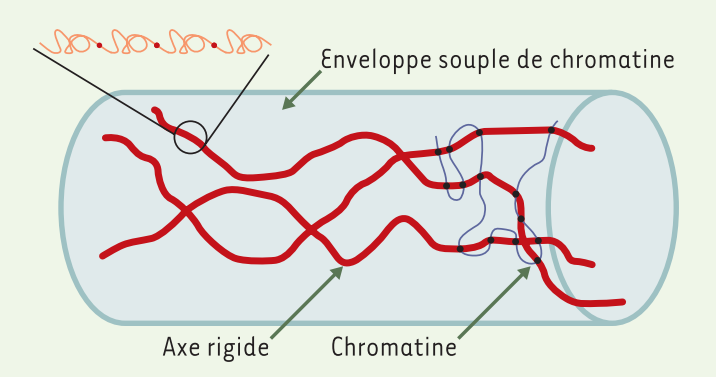

Figure 4. Structure du chromosome mitotique selon les mesures d'élasticité. Le chromosome est formé de quelques axes rigides de molécules titin-like entourés d'une enveloppe souple de chromatine (d'après [27]). des expériences de pontage, que l'actine se lie à l'ADN. Si l'actine peut avoir une fonction directe [32] de réorganisation de la chromatine, son rôle au niveau du centromère n'a pas pu être détecté. L'idée qu'un complexe actine-myosine pouvait avoir un rôle de structure, mais aussi un rôle fonctionnel réglable au niveau kinétochore-tubuline, est très controversée [33]. De même, alors que l'actine participe à la condensation de la chromatine chez le xénope, elle ne semble pas être un composant majeur de la métaphase.

\section{Conclusions}

Les chromosomes des eucaryotes subissent d'importantes modifications, une réorganisation physique et des mouvements programmés pendant la mitose. L'individualisation, la compaction des chromosomes répliqués (chromatides sœurs), puis leur ségrégation dans les cellules filles amènent le chromosome à modifier certaines de ses propriétés, comme son élasticité, et à résister aux forces des microtubules pour établir sa biorientation. La cohésion provient de ponts entre les chromatides sœurs, qui s'établissent tout le long du chromosome et empêchent une séparation prématurée des chromatides sœurs. Il est nécessaire de définir les protéines qui interviennent dans ce phénomène de tension qui s'oppose aux forces des microtubules. II est probable que le complexe cohésine et le complexe impliquant la titine cohabitent afin de donner aux chromosomes, par l'intermédiaire du centromère et des régions péricentriques, les meilleurs mouvements dans l'espace, tant longitudinaux qu'axiaux, dans une chronologie qui impliquerait les séquences $\alpha$-satellite (riches en AT) du centromère. $\diamond$

\section{SUMMARY}

\section{Centromere elasticity}

In addition to the role in the spindle apparatus and associated motors, the chromosome themselves play an important role in facilitating chromosome segregation. Sister chromatids are joined at the centromere through a protein complex called cohesin. Chromatids separation requires the degradation by separase of specific proteins acting as a glue to form the cohesin complex. This evolutionally complex is required for the establishment and maintenance of sister chromatids in a ring like structure. It is therefore a key question whether cohesin is indeed a main component of active centromere. Cohesin is insufficient to resist the splitting force exerted by microtubules until anaphase and must be renforced by cohesion provided by flanking DNA. The ring model suggests that cohesine might possess a conside- 
rable mobility when associated with chromatin. Observations demonstrate that the interior region of the centromere behaves as an elastic element. Chromosomes display remarkable elasticity, returning to their initial shape after being extended by up to 10 times. For larger deformations the thick filament is converted in thin filament which can be stretched six times before breaking. This article suggests an additional and novel role for the protein titin on chromosome structure and dynamic. Titine was identified as a chromosomal component and it was hypothesised that titin may provide elasticity to chromosome and resistance to chromosome breakages during mitosis. The elastic properties of purified titin correspond well to the elastic properties of chromosome in living cells. The deformability and bending rigidity are consistent with a model developed for titin elasticity. The association of the presence of cohesine ring and the activity of titin could be necessary for segregation. $\diamond$

\section{REMERCIEMENTS}

Je remercie Joël Martin (Groupe d'études des transcriptomes) pour son aide dans l'iconographie. Les travaux de l'équipe sont financés par l'Association pour la recherche sur le cancer (ARC).

\section{RÉFÉRENCES}

1. Pidoux AL, Allshire RC. Centromeres: getting a grip of chromosomes. Curr Opin Cell Biol 2000; 2 : 308-19.

2. Fukagawa T. Centromere DNA, proteins and kinetochore assembly in vertebrates cells. Chrom Res 2004; 12 : 557-67.

3. Roos UP. Light and electron microscopy of rat kangaroo cell in mitosis II kinetochore structure and function. Chromosoma 1973; 4: 195-220.

4. Mehes K, Bühler EM. Premature centromere division. Am J Med Genet $1995 ; 56: 76-9$.

5. Fitzgerald H, Archer SZ, Morris CM. Evidence for the repeat primary non disjunction of Chr-las the result of premature centromere division. Hum Genet 1986; 72 : 58-62.

6. Vig BK. Sequence of centromere separation: orderly separation of multicentric chromosomes in mouse cells. Chromosoma 1984; 90: 39-45.

7. Mehes G, Tarnok K, Pajor L, Mehes K. Objective analysis of centromere separation. Hum Genet 1996; 97 : 365-6.

8. Ghosh S, Paweletz N, Schroeter D. Failure of centromere separation leads to formation of diplochromosomes in next mitosis in okadaic acid treated HeLa cells. Cell Biol Int 1993; 17: 949-52.

9. Van Hooser AA, Mancini M, Allis CD, et al. The mammalian centromer: structural domains and the attenuation of chromatin modelling. FASEB J 1999; 13 (suppl 2): S216-20.

10. Rattner JB. The structure of mamalian centromere. BioEssays 1991; 13: 51-6.
11. Wheatley SP, Kandels-Lewis SE, Adams RR, et al. INCENP binds directly to tubulin and requires dynamic microtubules to target to the cleavage furrow. Exp Cell Res 2001; 262: 122-7.

12. Hirano T. SMC protein complexes and higher order chromosomes dynamics. Curr Opin Cell Biol 1998; 10 : 317-22.

13. Nicklas RB. Measurements of the force produced by the mitotic spindle in anaphase. J Cell Biol 1983; 987 : 542-8.

14. Rieder CL, Cole RW, Khodjakov A, Sluder G. The chekpoint delaying anaphase in reponse to chromosome monoorientation is mediated by an inhibitory signal produced by unattached kinetochore. J Cell Biol 1995; 130: $941-8$.

15. Shelby RD, Hahn KM, Sullivan KF. Dynamic elastic behavior of $\alpha$-satellite DNA domains visualized in situ in living human cells. J Cell Biol 1996; 135: 545-57.

16. Lan W, Zhang X, Kline-Smith SL, et al. Aurora B phosphorylates centromeric MCAK and regulates its localization and microtubule depolymerisation activity. Curr Biol 2004; 14: 273-86.

17. Yanagida $M$. Frontier questions about sister chromatid separation in anaphase. BioEssays 1995; 17: 519-26.

18. Koshland D, Guacci V. Sister chromatid cohesion: the beginning of a long and beautiful relationships. Curr Opin Cell Biol 2000; 12: 297-301.

19. Michaelis C, Ciosk R, Nasmyth K. Cohesins: chromosomal proteins that prevent premature separation of sister chromatids. Cell 1997; 91 : 35-45.

20. Hearing $\mathrm{CH}$, Nasmyth K. Building and breaking bridges between sisters chromatids. BioEssays 2003; 25: 1178-91.

21. Tanaka T, Fuchs J, Loidl J, Nasmyth K. Cohesin ensures bipolar attachment of microtubules to sister centromeres and resist their precocious separation. Nat Cell Biol 2000; 2: 492-5.

22. Campbell JL, Cohen-Fix 0 . Chromosome cohesion: ring around the sisters. Trends Biochem Sci 2002; $27:$ 492-5.

23. Bernard P, Allshire RC. Centromeres become unstuck without heterochromatin. Trends Cell Biol 2002; 12: 419-24.

24. Losada J, Hirano T. Intermolecular DNA interactions stimulated by the cohesion complex in vitro. Implications for sister chromatides cohesion. Curr Biol 2001; 11 : 268-72.

25. Claussen U, Mazur A, Rubtsov N. Chromosomes are highly elastic and can be stretched. Cytogenet Cell Genet 1994; 66: 120-5.

26. Houchmandzadeh B, Marko JF, Chatenay D, Libchaber L. Elasticity and structure of the eukaryotic chromosome studied by micromanipulation and micropipette aspiration. J Cell Biol 1997; 139: 1-12.

27. Houchmandzadeh B, Dimitrov $S$. Elasticity measurments show the existence of thin rigid cores inside mitotic chromosomes. J Cell Biol 1999; 145: 215-23.

28. Machado C, Andrew D. D-titin: a giant protein with dual roles in chromosomes and muscles. J Cell Biol 2000; 151: 639-52.

29. Machado C, Sunkel CE, Andrew D. Human antibodies reveal titin as chromosomal protein. J Cell Biol 1998; 141: 321-33.

30. Astier C, Labbe JP, Roustan C, Benyamin Y. Effect of different enzymatic treatments on the release of titin fragments from rabbit skeletal myofibrils. Biochem J 1993; 293: 731-4.

31. Astier C, Raynaud F, Lebart MC, et al. Binding of the native titin fragments to actin is regulated by PIP2. FEBS Lett 1998; 429: 95-8.

32. Runger-Brandle $\varepsilon$, Chapponnier C, Gabbiani G. Intranuclear injection of anti-actin antibodies into Xenopus oocytes bloks chromosomes condensation. Nature 1979; 282 : 320-1.

33. Silverman RV, Forer A. Evidence that actin and myosin are involved in the polarward flux of tubulin in metaphase kinetochore microtubules of crane-fly spermatocytes. J Cell Sci 2000 ; 113: 597-609. 